\title{
Topology, Locality, and Aharonov-Bohm Effect with Neutrons
}

\author{
Murray Peshkin \\ Physics Division, Argonne National Laboratory, Argonne, IL 60439-4843 USA \\ H. J. Lipkin \\ Department of Particle Physics, Weizmann Institute of Science, Rehovot 76100, Israel \\ and \\ School of Physics, Raymond and Beverly Sackler Faculty of Exact Sciences \\ Tel-Aviv University, Tel Aviv, Israel
}

\begin{abstract}
Recent neutron interferometry experiments have been interpreted as demonstrating a new topological phenomenon similar in principle to the usual Aharonov-Bohm (AB) effect, but with the neutron's magnetic moment replacing the electron's charge. We show that the new phenomenon, called Scalar AB (SAB) effect, follows from an ordinary local interaction, contrary to the usual $\mathrm{AB}$ effect, and we argue that the $\mathrm{SAB}$ effect is not a topological effect by any useful definition. We find that SAB actually measures an apparently novel spin autocorrelation whose operator equations of motion contain the local torque in the magnetic field. We note that the same remarks apply to the Aharonov-Casher effect.
\end{abstract}




\section{THE AHARONOV-BOHM EFFECT WITH ELECTRONS}

In the Aharonov-Bohm effect (AB), [1,2] idealized in Fig. 1, the motion of an electron in a Mach-Zehnder interferometer is influenced by electromagnetic fields even though the electron experiences no local, contemporaneous Maxwell field. That comes about because the Hamiltonian

$$
H=\frac{1}{2 m}\left(\boldsymbol{p}+\frac{e}{c} \boldsymbol{A}\right)^{2}-e V
$$

contains the gauge fields $\mathrm{V}$ and $\mathbf{A}$, which have nonvanishing values at some points in the domain of the electron's position r. For AB effect, we can ignore the electron's spin. Then the operator equations of motion for the only observables are

$$
\dot{\boldsymbol{r}}=\boldsymbol{v} \quad m \dot{\boldsymbol{v}}=0,
$$

those of a free particle, containing no electromagnetic fields. However, in quantum mechanics the equations of motion alone do not determine the dynamics. In the magnetic $\mathrm{AB}$ effect (Fig. 1a), the partial waves in the two arms of the interferometer acquire a relative phase shift $\delta \phi$ given by

$$
\delta \phi=\frac{e}{\hbar c} \oint A \cdot d \boldsymbol{r}=\frac{e \Phi}{\hbar c}
$$

where $\Phi$ is the flux through the solenoid.

In the electric $\mathrm{AB}$ effect (Fig. 1b), the two arms of the interferometer carry the electron through conducting cylinders that shield the electron from an electric field. While the split wave packet is deep within one cylinder or the other, potentials $V_{1}$ and $V_{2}$ are applied to the two cylinders. That causes a relative phase shift given by

$$
\delta \phi=\frac{e}{\hbar}(\Delta V) \tau
$$

where $\Delta V=V_{1}-V_{2}$ and $\tau$ is the length of the time interval during which $\Delta V$ is different from zero.

In both cases, the relative phase shift is measured by the outbound intensities

$$
I_{1}=I \cos ^{2}(d \phi) \quad I_{2}=I \sin ^{2}(d \phi) .
$$

The AB effect is nonlocal in that the electron experiences no force and exchanges no momentum, energy, or angular momentum with the electromagnetic field; and in that the Hamiltonian, the equations of motion, and the commutation relations involve no local contemporaneous Maxwell field at the electron's position.

$\mathrm{AB}$ is a topological effect in that it requires the electron to be confined to a multiplyconnected region and in that there is no objective way to relate a phase shift to any particular place or to either arm of the interferometer. The phase shift between any two Feynman amplitudes depends only upon the difference between the topological winding numbers $n$ of their paths.

$$
\delta \phi=(\delta n) \frac{e \Phi}{\hbar c} .
$$


The relative phase shift depends upon an integral whose integrand is not gauge invariant and not observable. The only gauge-invariant observable is the integral of this integrand over a closed path, and its value is proportional to the magnetic flux enclosed by this path. This effect is manifestly nonlocal, since its value depends upon a physical quantity in a region outside the domain of integration. It is topological in the sense that it depends only upon the topology of the path with reference to the enclosed magnetic flux. In an interferometer, the winding numbers of the two arms differ by unity. The general role of the winding numbers is more obvious in the magnetic scattering geometry, illustrated in Fig. 2. The differences in phase shift between different paths are gauge invariant, but no measurable phase shift can be assigned to any one path because $\int \boldsymbol{A} \cdot d \boldsymbol{r}$ along any one path depends upon the choice of gauge. The same is true of the electric $\mathrm{AB}$ effect. The potential difference $\Delta V$ is gauge invariant, but the potential $\mathrm{V}$ on one of the cylinders can be given any value by choice of gauge. Therefore there is no objective way to associate the phase shift with one arm of the interferometer or the other.

\section{THE SCALAR AHARONOV-BOHM EFFECT WITH POLARIZED NEUTRONS}

In a recent series of experiments, Allman et al. [3, [1] passed unpolarized neutrons through a Mach-Zehnder interferometer one arm of which traversed a magnetic field $\mathbf{B}$, as shown schematically in Fig. 3. The intensities of the two outbound beams were observed to obey Eq. (1.5), where now the relative phase shift is given by

$$
\delta \phi=\frac{\mu}{\hbar} B \tau,
$$

where $\mu$ is the neutron's magnetic moment, $\mathrm{B}$ is the magnetic field strength, and $\tau$ is the time spent in the magnetic field. The experimenters interpreted their results as demonstrating a new topological effect which they named Scalar Aharonov-Bohm effect (SAB). That claim was refuted by one of us, [5] who pointed to ambiguities introduced by the use of unpolarized neutrons. (The same point had been made earlier by Zeilinger, [6] and the meaning of this kind of experiment was also discussed by Anandan. [7,8])

Here we will analyze the ideal SAB experiment, also illustrated by Fig. 3, in which: the neutron is to be polarized with $\sigma_{z}=+1$, where the $z$ direction is that of the magnetic field, assumed to be spatially uniform; $\mathrm{B}(\mathrm{t})$ vanishes except during a time interval of length $\tau$ when it has the value $\mathrm{B}$; and the neutron is assumed to be in the magnetic field region throughout the time interval $t$ so that it never experiences a field gradient. The relation of the $z$ direction to the plane of Fig. 3 is immaterial. The purposes of this analysis are to show that using polarized neutrons will not help and to explain how SAB differs in principle from $\mathrm{AB}$.

In $\mathrm{SAB}$, the Hamiltonian

$$
H=\frac{\boldsymbol{p}^{2}}{2 m}-\mu \boldsymbol{\sigma} \cdot \boldsymbol{B}(t)
$$

contains the Maxwell field $\boldsymbol{B}$, in contrast to AB, where the Hamiltonian (1.1) contains only the gauge fields. The operator equations of motion 


$$
\frac{\hbar}{2} \dot{\boldsymbol{\sigma}}=\mu \boldsymbol{\sigma} \times \boldsymbol{B}(t)
$$

contain the local contemporaneous Maxwell field, in contrast to AB, where no electromagnetic field enters the equation of motion of any measurable quantity.

However, it is argued that $\mathrm{SAB}$ resembles the electric $\mathrm{AB}$ affect $(\mathrm{EAB})$ in that role of $\boldsymbol{B}$ in the SAB Hamiltonian (2.2) is very much that of a potential acting on the magnetic moment and in that no force acts on the neutron. Also, the consequences of Eq. (2.3) are possibly uncertain because $\left\langle\sigma_{x}(t)\right\rangle=\left\langle\sigma_{y}(t)\right\rangle=0$ in a state with $\sigma_{z}=+1$. In terms of the Schroedinger equation, one may replace $\sigma_{z}$ by the number +1 in the Hamiltonian (2.2) so that it becomes

$$
H=\frac{\boldsymbol{p}^{2}}{2 m}-\mu B(t)
$$

and restrict the Hilbert space to what appears as a one-component wave function with no dynamical variables other that $\boldsymbol{x}$ and $\boldsymbol{v}$. Then the mathematical analogy with EAB is complete and one has the illusion [3, 4] that SAB is a nonlocal, topological effect in the same sense as is EAB.

That reasoning gets the correct phase shift but it leads to an incorrect interpretation of the experiment. In SAB, the relative phase shift depends upon an integral whose integrand is locally gauge invariant and observable at every point in the path of the neutron. The integrand is proportional to the magnetic field directly in the path of the neutron and does not depend upon a physical quantity in a region outside that path. SAB does not have the same topological character as AB, because the SAB phase shift depends upon the local field along the path and not upon any winding number expressing the topology of a path around a region in which the particle does not move. The operator equations of motion do involve the local, contemporaneous Maxwell field.

Moreover, in quantum mechanics, the spin is a dynamical variable and it cannot simply be replaced by a number. The right hand side of Eq. (2.3) is a torque $\boldsymbol{L}$ on the neutron whose expectation value vanishes at all times but whose fluctuations do not vanish.

$$
\begin{aligned}
& \left\langle L_{x}\right\rangle=\left\langle L_{y}\right\rangle=0 \\
& \left\langle L_{x}^{2}\right\rangle=\left\langle L_{y}^{2}\right\rangle=(m B)^{2}
\end{aligned}
$$

Then an equal and opposite angular momentum must be transmitted to the local electromagnetic field, again with zero expectation but with fluctuations correlated with those of the neutron's angular momentum so that the total angular momentum is conserved. Those field angular momentum fluctuations are not observable by a measurement on the field in the limit of a classical field, but they are observable in principle in a finite field.

The effect of the torque on the neutron is exposed by considering the spin autocorrelation operators

$$
\begin{aligned}
C(t) & =\frac{1}{4}\left[\sigma_{x}(0) \sigma_{x}(t)+\sigma_{y}(0) \sigma_{y}(t)+h . c .\right] \\
S(t) & =\frac{1}{4}\left[\sigma_{x}(0) \sigma_{y}(t)-\sigma_{y}(0) \sigma_{x}(t)+h . c .\right]
\end{aligned}
$$

These are Hermitean operators, measurable in principle, and they commute with so there is no question about their significance in a state of definite $\sigma_{z}$. Their equations of motion, 


$$
\begin{aligned}
\dot{C}(t) & =\frac{2 \mu B}{\hbar} S(t) \\
\dot{S}(t) & =\frac{2 \mu B}{\hbar} C(t),
\end{aligned}
$$

contain the local contemporaneous Maxwell field and the solutions are given by

$$
\begin{aligned}
& C(t)=\cos (\omega t) \\
& S(t)=-\sin (\omega t),
\end{aligned}
$$

where

$$
\omega=2 \mu B / \hbar \text {. }
$$

These spin correlation operators cannot be described classically for spin $1 / 2$, but they can be described simply in the context of the usual semiclassical vector model. There, the vectors $\boldsymbol{\sigma}(0)$ and $\boldsymbol{\sigma}(t)$ are depicted as precessing on a cone with random phase so that their projections on the xy plane vanish on the average. Equations $(2.7,2.8)$ show that the relative angle $\vartheta(t)=\omega t$ between the two projections is changed by the action of the local torque.

When the two partial waves merge at the final mirror of the interferometer in Fig. 3, their spin correlation angle is

$$
\vartheta(\tau)=\omega \tau=2 \delta \phi
$$

The intensities in the two outgoing beams are of course given by the same Eqs. (1.5). However, now the effect has been described as the measurement of a spin correlation. The factor 2 in Eq. (2.10) is the usual factor for rotations of spin $1 / 2$.

None of this is really surprising from either a classical or a quantum mechanical point of view. A spinning particle is represented classically as a symmetric rotor whose angular momentum precesses in a magnetic field. The precession frequency $\omega$ is independent of the angle between the rotation axis and the magnetic field. That is why the spin autocorrelations are independent of the spin state in Eqs. (2.8). Classically, the only exceptions are the two states wherein the spin points exactly in the $+z$ or the $-z$ direction, a set of measure zero for which the $\mathrm{x}$ and $\mathrm{y}$ components vanish and the precession frequency has no meaning. However, if one defines the precession frequency by any limiting process, it again has the value $\omega$. In quantum mechanics only the expectation values of $\sigma_{x}$ and $\sigma_{y}$ vanish. Their fluctuations are large, equal in magnitude to $\sigma_{z}$. In quantum mechanics, the local magnetic field separates the energies of the two states of definite $\sigma_{z}$ and that energy separation gives rise to the precession of $\sigma_{x}$ and $\sigma_{y}$ which becomes visible in the spin autocorrelation functions.

\section{CONCLUSIONS}

The Scalar Aharonov-Bohm effect has been described as the ordinary action of a magnetic field on the magnetic moment of the neutron, causing the neutron to precess in the ordinary way. The return torque transmits angular momentum to the local contemporaneous magnetic field in the ordinary way. Locality in the sense of Faraday and Maxwell is preserved to the extent that it ever is in quantum mechanics. 
We have identified measurable dynamical variables, the spin autocorrelation operators, whose operator equations of motion obey the classical laws. The conventional semiclassical vector model shows exactly how the torque in the magnetic field acts on the spin autocorrelation.

$\mathrm{SAB}$ is not a topological effect in the same sense as is the $\mathrm{AB}$ effect, in spite of the mathematical similarity of SAB and electric $\mathrm{AB}$ effect. In SAB, we know exactly where the neutron experienced the torque that changed the outcome of the experiment, and no gauge transformation can obscure that information.

Allman et al. [1] defined a topological effect as one in which the relative phase shift $\delta \phi$ is independent of the energy of the neutron. That criterion was justified by a result of Zeilinger [6.9], who however showed only that the energy independence is a necessary condition for a force-free effect.

The trouble with using that criterion in the present context can be seen by considering a problem in which the magnetic field in one arm of the interferometer is replaced by an optical phase shifter whose index of refraction is made to depend upon the time and to differ from unity only during the time the neutron is inside some box, for instance by pumping a refractive gas in and out. In principle, the phase shift can be made independent of the energy over the experimental range. No electromagnetic field is involved. The energy-independence criterion would describe the influence of that phase shifter as a topological effect.

We have chosen to discuss the Aharonov-Bohm effect on the magnetic moment of a spin$1 / 2$ particle in terms of the SAB effect because of the experimental interest in that example. However the discussion is identical for the Aharonov-Casher $(\mathrm{AC})$ effect [10]. In $\mathrm{AC}$, a neutron with $\sigma_{z}=+1$ traverses an external electric field in the $x y$ plane. In an adequate approximation, the AC Hamiltonian is given by Eq.J (2.2), where now $\mathbf{B}$ is the magnetic field in the rest frame of the neutron, given by

$$
\boldsymbol{B}=\frac{\boldsymbol{p}}{m c} \times \boldsymbol{E}(\boldsymbol{r}) .
$$

For a neutron whose velocity is confined to the $x y$ plane, $\boldsymbol{B}$ points in the $z$ direction and interference effects not ascribable to forces, like those in SAB, are predicted. However, the torques, spin autocorrelations, and angular momentum exchange with the local Maxwell field appear to be the same as in SAB, so it follows that AC, like SAB, is neither a nonlocal nor a topological effect.

The basic physics underlying our argument is in fact very simple. The spin of a neutron precesses in an external magnetic field as a result of the local interaction of the neutron magnetic moment with the field. This precession has been observed in many experiments. It is conjectured that such precession is absent when the neutron spin is exactly in the directions of the field and the components of the spin normal to the field vanish exactly; e.g. for a field in the z-direction the spin components satisfy the condition

$$
\sigma_{x}=\sigma_{y}=0
$$

However this condition can be satisfied in classical mechanics only for a set of states of measure zero. In quantum mechanics this condition cannot be satisfied at all, since the operators $\sigma_{x}$ and $\sigma_{y}$ do not commute with one another and furthermore do not have an allowed zero eigenvalue. 
The expectation values of $\sigma_{x}$ and $\sigma_{y}$ do indeed vanish when a neutron is "polarized in the z-direction"; i.e. when it is in an eigenstate of $\sigma_{z}$. However, this only means that their average value vanishes. We have shown here that the precession in the magnetic field of the spin components normal to the field is still observable, even when the neutron is so-called "polarized in the direction of the field". This precession is in fact observed experimentally in the $\mathrm{AC}$ and $\mathrm{SAB}$ effects. We have pointed out a marked difference between the topology and locality which characterize the $\mathrm{AB}$ and the analogous considerations in $\mathrm{AC}$ and SAB. Instead the $\mathrm{SAB}$ experiment provides evidence that the normal components of the neutron spin do indeed precess with the normal precession frequency in an external magnetic field, even though the expectation values of these normal components vanish. The precession is expressed formally by spin autocorrelation functions.

This work is supported in part by the U. S. Department of Energy, Nuclear Physics Division, under contract W-31-109-ENG-38. 


\section{REFERENCES}

[1] Y. Aharonov and D. Bohm, Phys. Rev. 115, 485 (1959).

[2] M. Peshkin and A. Tonomura, "The Aharonov-Bohm Effect", Lecture Notes in Physics No. 340 (Springer-Verlag 1989).

[3] B. E. Allman et al., Phys. Rev. Lett. 68, 2409 (1992).

[4] B. E. Allman et al., Phys. Rev. A 48, 1799 (1993).

[5] M. Peshkin, Phys. Rev. Lett. 69, 2017 (1992).

[6] A. Zeilinger in "Fundamental Aspects of Quantum Theory", NATO ASI Series B, Vol. 144, eds. V. Gorini and A. Frigerio (1986) pp. 331.

[7] J. Anandan, Phys. Rev. Lett. 24, 1660 (1982).

[8] J. Anandan in "Proceedings of the 3rd International Symposium Foundations of Quantum Mechanics in the Light of New Technology", Physical Society of Japan, eds. S. Kobayashi, H. Ezawa, Y. Murayama, and S. Nomura (1989) pp. 98.

[9] G. Badurek et al., Phys. Rev. Lett. 71, 307 (1993).

[10] Y. Aharonov and A. Casher, Phys. Rev. Lett. 53, 319 (1984); see also A. S. Goldhaber, Phys. Rev. Lett. 62, 482 (1989). 


\section{FIGURES}

FIG. 1. (a) Magnetic Aharonov-Bohm effect. The shaded area is a solenoid.

(b) Electric Aharonov-Bohm effect.

FIG. 2. Three Feynman paths from $X_{1}$ to $X_{2}$ with winding numbers.

FIG. 3. Interferometer for polarized neutrons. The shaded area is the magnetic field region. 
This figure "fig1-2.png" is available in "png" format from: http://arxiv.org/ps/quant-ph/9501012v1 